\title{
Ultra-high speed reconfigurable microwave and radio frequency photonic intensity differentiators using Kerr frequency optical microcombs
}

David Moss ( $\sim$ dmoss@swin.edu.au)

Swinburne University of Technology https://orcid.org/0000-0001-5195-1744

\section{Research Article}

Keywords: Microwave photonic, signal channelization, integrated optical frequency comb

Posted Date: November 8th, 2021

DOI: https://doi.org/10.21203/rs.3.rs-1054757/v1

License: (c) (1) This work is licensed under a Creative Commons Attribution 4.0 International License.

Read Full License 


\section{Abstract}

We propose and experimentally demonstrate a microwave photonic intensity differentiator based on a Kerr optical comb generated by a compact integrated micro-ring resonator (MRR). The on-chip Kerr optical comb, containing a large number of comb lines, serves as a high-performance multi-wavelength source for the transversal filter, which will greatly reduce the cost, size, and complexity of the system. Moreover, owing to the compactness of the integrated MRR, up to $200-\mathrm{GHz}$ frequency spacing of the Kerr optical comb can be achieved, enabling a potential operation bandwidth of over $100 \mathrm{GHz}$. By programming and shaping individual comb lines according to the calculated tap weights, a reconfigurable intensity differentiator with variable differentiation orders can be realized. The operation principle is theoretically analyzed, and experimental demonstrations of first-order, second-order, and thirdorder differentiation functions based on the principle are presented. The radio frequency (RF) amplitude and phase responses of multi-order intensity differentiations are characterized, and system demonstrations of real-time differentiations for Gaussian input signal are also performed. The experimental results show good agreement with theory, confirming the effectiveness of our approach.

\section{Introduction}

With the ever-increasing demand for processing speed and throughput in modern communication systems, optical information processing technologies have attracted great interest due to their advantages in overcoming the intrinsic bandwidth bottleneck of electronic processing [1-6]. As one of the basic building blocks in optical signal processing and computing systems [7], photonic differentiators are a key requirement in high-speed signal analyzing, waveform shaping, pulse generation, and system control [8-10].

To implement photonic differentiators, a number of schemes have been proposed, which can be classified into two categories, namely, field differentiators and intensity differentiators [11]. Field differentiators based on apodized fibre Bragg gratings $[8,10,12]$ and integrated silicon photonic devices [13-16] have been recently demonstrated. These types of differentiators yield the derivative of a complex optical field, having the ability to shape ultra-short optical pulses that could find applications in optical pulse generation and advanced coding [17-19]. On the other hand, some other applications such as ultrawideband (UWB) frequency generation, radio frequency (RF) measurement, and RF filters, require intensity differentiators that could provide the derivative of the temporal intensity profiles associated with RF signals [20-22]. A photonic intensity differentiator based on a dual-drive Mach-Zehnder modulator (DDMZM) together with a RF delay line was reported in Ref. [11], but the processing speed was intrinsically limited by the operation bandwidth of the RF delay line. Photonic intensity differentiators based on semiconductor optical amplifiers (SOAs) and optical filters (OFs) have also been reported [2324], featuring high processing speeds up to $40-\mathrm{Gb} / \mathrm{s}$. This approach, however, works only for a fixed differentiation order and lacks reconfigurability, whereas processing systems with variable differentiation orders are desired in practical applications, in order to meet diverse computing requirements. To implement highly reconfigurable intensity differentiators, transversal filter schemes based on discrete 
microwave photonic delay-lines have been investigated $[9,25]$. However, these approaches had limitations of one form or another, such as the need of generating the taps using discrete laser arrays, thus significantly increasing the system cost and complexity.

In this paper, a reconfigurable microwave photonic intensity differentiator based on an integrated Kerr comb source is proposed and experimentally demonstrated. By employing an on-chip nonlinear MRR, we generate a broadband Kerr comb with a large number of comb lines and use it as a high-quality multiwavelength source for the transversal filter. Moreover, the large frequency spacing of the integrated Kerr comb also yields an increased Nyquist zone, thus leading to a potential operation bandwidth of over 100 $\mathrm{GHz}$, well beyond the processing bandwidth of electronic devices. By programming and shaping the power of individual comb lines according to corresponding tap weights [26-27], reconfigurable intensity differentiators with variable differentiation orders can be achieved. We present a detailed analysis of the operation principle, and perform experimental demonstrations of first-, second-, and third-order intensity differentiations using the proposed device. The RF amplitude and phase responses of the intensity differentiator are experimentally characterized, and system demonstrations of real-time differentiations for Gaussian input pulses are also carried out. The experimental results are consistent with theory, verifying the feasibility of our approach as a solution to high-speed reconfigurable microwave photonic intensity differentiators.

\section{li. Operation Principle}

Based on the classical theory of signals and systems [28], the spectral transfer function of an $\mathrm{N}$-th order temporal differentiator can be expressed as

\section{$H(\omega) \propto(j \omega)^{N}$,}

where $j=\sqrt{-1}, \omega$ is the angular frequency, and $N$ is the differentiation order. According to the above transfer function, the amplitude response of a temporal differentiator is proportional to $|\omega|^{N}$, while the phase response has a linear profile and a zero or $\pi$ jump at zero frequency for $N$ even and odd, respectively. The ideal RF amplitude and phase responses of first-order, second-order, and third-order microwave differentiators are shown in Figs. 1(a)-(c), respectively.

In this paper, we employ a versatile approach towards the implementation of microwave photonic differentiators based on transversal filters, where a finite set of delayed and weighted replicas of the input RF signal are produced in the optical domain and combined upon detection [29-31]. The transfer function of a typical transversal filter can be described as

$$
H(\omega)=\sum_{n=0}^{M-1} a_{n} e^{-j \omega n T}
$$


where $M$ is the number of taps, $a_{n}$ is the tap coefficient of the $n$-th tap, and $T$ is the time delay between adjacent taps. It should be noted that the differentiator design based on Eq. (2) is an intensity differentiator, i.e., the output RF signal after being combined upon detection yields an exact differentiation of the input RF signal, in contrast to field differentiators that yield the derivative of a complex optical field $[8,10,12-16]$.

To implement the temporal differentiator in Eq. (1), we calculate the tap coefficients in Eq. (2) based on the Remez algorithm [32]. The corresponding amplitude and phase responses of the first-, second-, and third-order differentiators as a function of the numbers of taps are plotted in Figs. 1(a)-(c). When the number of taps is increased, it is clear that the discrepancies between the amplitude responses of the transversal filters and the ideal differentiators are improved for all the three orders, whereas the phase response of the transversal filters is identical to that of the ideal differentiators regardless of the number of taps. To quantitatively analyze the discrepancies in the amplitude responses, we further calculate the root mean square errors (RMSEs) for the first-, second-, and third-order differentiators as a function of the number of taps, as shown in Fig. 1(d). One can see that the RMSE is inversely proportional to the number of taps, as reasonably expected. In particular, we note that when the number of taps increases, the RMSE decreases dramatically for a small number of taps, and then decreases more gradually as the number of taps becomes larger.

Figure 2 shows the schematic illustration of the reconfigurable microwave photonic intensity differentiator. It consists of two main blocks: one is a Kerr comb generation module based on a nonlinear MRR and the other is a transversal filter module for reconfigurable intensity differentiation. In the first module, the continuous-wave (CW) light from a tunable laser source (TLS) is amplified by an erbiumdoped fibre amplifier (EDFA) followed by a tunable optical bandpass filter (BPF) to suppress the amplified spontaneous emission (ASE) noise. A polarization controller (PC) is inserted before the nonlinear MRR to make sure that the polarization state matches the desired coupled mode. When the wavelength of the $\mathrm{CW}$ light is tuned to a resonance of the nonlinear MRR and the light power is large enough for sufficient parametric gain, the optical parametric oscillation (OPO) process in the nonlinear MRR can be initiated, which generates a Kerr optical comb with nearly equal line spacing [33-34]. The nonlinear MRR is mounted on a highly precise temperature controlled stage (TCS) to avoid resonance drifts and maintain the wavelength alignment of the resonances to the CW light. Owing to the compact size and ultra-high quality factor of the nonlinear MRR, the generated Kerr comb provides a large number of wavelength channels with narrow linewidths for the subsequent transversal filter module. As compared with conventional intensity differentiators based on laser diode arrays, the cost, size and complexity can be greatly reduced. The generated Kerr comb then is directed to the second module. After being amplified by another EDFA, the Kerr comb is processed by a waveshaper to get weighted taps according to the coefficients calculated by means of the Remez algorithm. Considering that the generated Kerr comb is not flat, a real-time feedback control path is introduced to read and shape the comb lines' power accurately. A $2 \times 2$ balanced Mach-Zehnder modulator (MZM) is employed to generate replicas of the input RF signal. When the MZM is quadrature-biased, it could simultaneously modulate the input RF signal on both positive and negative slopes, thus yielding modulated signals with opposite phases and tap 
coefficients with opposite algebraic signs. After being modulated, the tapped signals from one output of the MZM are delayed by a dispersive fibre. The time delay between adjacent taps is co-decided by the frequency spacing of the employed comb source and the dispersion accumulated in the fibre. Finally, the weighted and delayed taps are combined upon detection and converted back into RF signals to form the differentiation output.

It is worth mentioning that due to the intrinsic advantages of transversal filters, our scheme features a high degree of reconfigurability in terms of processing functions and operation bandwidth, thus offering a reconfigurable platform for diverse microwave photonic computing functions. By simply programming the waveshaper to shape the comb lines according to the corresponding tap coefficients, our scheme can also apply to other computing functions such as Hilbert transforms and differential equation solving [35-36]. The operation bandwidth can also be changed by adjusting the time delay between adjacent taps or employing specific tap coefficients. An increased operation bandwidth can be achieved by simply employing a dispersive fibre with shorter length. The operation bandwidth is fundamentally limited by the Nyquist zone, which is determined by the comb spacing. In our case, the frequency spacing of the Kerr comb generated by the nonlinear MRR reaches $200 \mathrm{GHz}$, thus leading to a potential operation bandwidth of over $100 \mathrm{GHz}$.

\section{lii. Experimental Results}

In our experiment, as Fig. 3 (a) shows, the nonlinear MRR used to generate the Kerr comb was fabricated on a high-index doped silica glass (Hydex) platform using CMOS compatible fabrication processes [33]. First, high-index ( $n=1.7$ ) doped silica glass films were deposited using standard plasma enhanced chemical vapour deposition (PECVD), then photolithography and reactive ion etching (RIE) were employed to form waveguides with exceptionally low surface roughness. Finally, the waveguides were buried in a lower index overcladding. The radius of the Hydex MRR is $\sim 135 \mu \mathrm{m}$. The compact integrated MRR has a large free spectral range (FSR) of $\sim 1.6 \mathrm{~nm}$, i.e., $\sim 200 \mathrm{GHz}$. Such a large FSR enables an increased Nyquist zone of $\sim 100 \mathrm{GHz}$, which is challenging for mode-locked lasers and externallymodulated comb sources [37-38]. The advantages of the Hydex platform for nonlinear OPO include ultra-low linear loss $\left(\sim 0.06 \mathrm{~dB} \rrbracket \mathrm{cm}^{-1}\right)$, a moderate nonlinearity parameter $\left(\sim 233 \mathrm{~W}^{-1} \triangle \mathrm{km}^{-1}\right)$, and in particular a negligible nonlinear loss up to extremely high intensities $\left(\sim 25 \mathrm{GW} \llbracket \mathrm{cm}^{-2}\right)$ [33-34]. After packaging the input and output ports of the device with fibre pigtails, the total insertion loss is $\sim 3.5 \mathrm{~dB}$. A scanning electron microscope (SEM) image of the cross-section of the Hydex MRR before depositing the $\mathrm{SiO}_{2}$ upper cladding is shown in Fig. 3 (b). By boosting the power of the CW light from the TLS via an EDFA and adjusting the polarization state, multiple mode-spaced combs were first generated, in which the primary spacing was determined by the parametric gain. When the parametric gain lobes became broad enough, secondary comb lines with a spacing equal to the FSR of the Hydex MRR were generated via either degenerate or non-degenerate four wave mixing (FWM). In our experiment, the power threshold for the generation of secondary comb lines was $\sim 500 \mathrm{~mW}$. 
The resulting Type II Kerr optical comb [39] as shown in Fig. 4(a) is over 200-nm wide, and flat over 32 $\mathrm{nm}$. Since the generated comb only served as a multi-wavelength source for the subsequent transversal filter in which the optical power from different taps was detected incoherently by the photodiode, the coherence of the comb was not crucial and the proposed differentiator was able to work under relatively incoherent conditions. In the experiment, the numbers of taps used for first-, second-, and third-order differentiation demonstrations were 8,6 , and 6 , respectively. The choice of these numbers was made mainly by considering the power dynamic range of the comb lines, i.e., the difference between the maximum power of the generated comb lines and the power associated with the noise floor. The power dynamic range was determined by the EDFA before waveshaping, and in our case, it was $\sim 30 \mathrm{~dB}$. An increased number of taps requires a broader power dynamic range, which can be achieved by using a

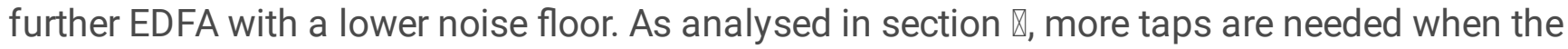
differentiationorder increases, and for a fixed number of taps, increasing the order of differentiation also increases the required power dynamic range. In order to get better performance with a limited number of taps, we decreased the operation bandwidth of the second- and third-order differentiators to half of the transversal filter's Nyquist frequency when designing the response function with the Remez algorithm. It should be noted that the actual bandwidth of the differentiator is not limited by this design since the FSR of the transversal filter can be enlarged. The calculated tap coefficients for first-, second-, and third-order differentiation are listed in Table I. The selected comb lines of the generated optical comb were processed by the waveshaper based on these coefficients. Considering that the generated Kerr comb was not flat, we adopted a real-time feedback control path to increase the accuracy of comb shaping. The comb lines' power was first detected by an optical spectrum analyzer (OSA) and compared with the ideal tap weights, and then an error signal was generated and fed back into the waveshaper to calibrate the system and achieve accurate comb processing. The shaped optical combs are shown in Figs. 4(b)-(d). A good match between the measured comb lines' power (red solid line) and the calculated ideal tap weights (green cross) was achieved, indicating that the comb lines were successfully shaped. They were then divided into two parts according to the algebraic sign of the tap coefficients andfed into the $2 \times 2$ balanced MZM biased at quadrature. The modulated signal after the MZM was propagated through $\sim 2.122-\mathrm{km}$ single mode (dispersive) fibre (SMF). The dispersion of the SMF is $\sim 17.4 \mathrm{ps} /(\mathrm{nm} \otimes \mathrm{km})$, which corresponds to a time delay of $\sim 59$ ps between adjacent taps and yields an effective FSR of $\sim 16.9 \mathrm{GHz}$ in the RF response spectrum.

After the weighted and delayed taps were combined upon detection, the RF responses for different differentiation orders were characterized by a vector network analyser (VNA, Anritsu 37369A). Figures. $5(a-i),(b-i)$, and (c-i) show the measured and simulated amplitude responses of the first-, second-, and third-order intensity differentiators, respectively. The corresponding phase responses are depicted in Figs. 5(a-ii), (b-ii), and (c-ii). It can be seen that all the three differentiators feature the responses expected from ideal differentiation. The FSR of the RF response spectra is $\sim 16.9 \mathrm{GHz}$, which is consistent with the time delay between adjacent taps, as calculated before measurement. Note that by adjusting the FSRof the proposed transversal filter through the dispersive fibre or by programming the tap coefficients, a variable 
operation bandwidth for the intensity differentiator can be achieved, which is advantageous for meeting diverse requirements in operation bandwidth.

We also performed system demonstrations of real-time signal differentiation for Gaussian input pulses with a full-width at half maximum (FWHM) of $\sim 0.12 \mathrm{~ns}$, generated by an arbitrary waveform generator (AWG, KEYSIGHT M9505A), as shown in Fig. 6(a). The waveform of the output signals after differentiation are shown in Figs. 6 (b)-(d) (blue solid curves). They were recorded by means of a highspeed real-time oscilloscope (KEYSIGHT DSOZ504A Infiniium). For comparison, we also depict the ideal differentiation results, as shown in Figs. 6 (b)-(d) (red dashed curves). The practical Gaussian pulse in Fig. 6(a) is used as the input RF signal for the simulation. One can see that the measured curves closely match their theoretical counterparts, indicating good agreement between experimental results and theory. Unlike the field differentiators in Refs. $[8,10,12-16]$, temporal derivatives of intensity profiles can be observed, indicating that intensity differentiation was successfully achieved. For the first-, second-, and third-order differentiators, the calculated RMSE between the measured and the theoretical curves are $\sim 4.15 \%, \sim 6.38 \%$, and $\sim 7.24 \%$, respectively.

To further investigate the imperfections associated with the experimental results, we employed commercial software (VPI Photonics) to simulate the RF amplitude and phase responses of the various differentiators by considering the tap weight error during the comb shaping, the chirp induced by the $M Z M$, and the third-order dispersion (TOD) of the fibre. Based on the measured error signal from the feedback control path and the empirical chirp value in previous experiments [35], the tap weight error, chirp coefficient, and TOD in our simulation were set to be $0.5 \mathrm{~dB}, 0.5$, and $0.083 \mathrm{ps} /\left(\mathrm{nm}^{2} \llbracket \mathrm{km}\right)$, respectively. The simulated amplitude and phase responses are plotted in Figs. 5 (a)-(c). One can see that after incorporating these two effects, the simulated RF responses fit more closely to the experimental results, thus confirming that the degradation of the RF responses can be attributed to both of these effects. By employing a low-chirped MZM, further increasing the resolution of the feedback control path, and compensating the TOD, we can anticipate improved performance of the differentiators.

\section{Iv. Discussion}

All-optical signal processing based on nonlinear optics has proven to be extremely powerful, particularly when implemented in photonic integrated circuits based on highly nonlinear materials such as silicon [40-43]. All optical signal processing functions include all-optical logic [44], demultiplexing at ultra-high bit rates from $160 \mathrm{~Gb} / \mathrm{s}$ [45] to over $1 \mathrm{~Tb} / \mathrm{s}$ [46], optical performance monitoring (OPM) using slow light $[47,48]$, all-optical regeneration $[49,50]$, and others [51-56]. Complementary metal oxide semiconductor (CMOS) compatible platforms are centrosymmetric and so the $2 \mathrm{rd}$ order nonlinear response is zero. Hence, nonlinear devices in these platforms have been based on the 3rd order nonlinear susceptibility including third harmonic generation [51, 57-61] and the Kerr nonlinearity $\left(n_{2}\right)$ [40-42]. The efficiency of Kerr nonlinearity based all-optical devices depends on the waveguide nonlinear parameter $(\mathrm{Y})$. Although silicon-on-insulator nanowire devices can achieve extremely high nonlinearities ( $\mathrm{Y}$ ), they suffer from high nonlinear optical losses due to two-photon absorption (TPA) and the resulting generated free carriers [42]. 
Even if the free carriers are swept out by $\mathrm{p}-\mathrm{i}-\mathrm{n}$ junctions, silicon's relatively poor intrinsic nonlinear figure of merit (FOM $=\mathrm{n} 2 /(\beta \lambda)$, where $\beta$ is the TPA and $\lambda$ the wavelength) of around 0.3 in the telecom band is too low to achieve good performance. While TPA can be turned to advantage for some all-optical functions [62-64], for the most part, silicon's low FOM in the telecom band is a limitation. This has motivated research on a range of alternate nonlinear platforms including chalcogenide glasses [65-74]. However, while offering many advantages, these platforms are not compatible with CMOS processing the basis of the silicon computer chip industry.

In 2007/8 new CMOS compatible platforms for nonlinear optics were reported that exhibited extremely low two-photon absorption in the telecommunications wavelength band. These included silicon nitride $[75,76]$ as well as high-index doped silica glass (Hydex) [77-87], similar in its optical properties to silicon oxynitride. In addition to negligible nonlinear absorption, these platforms displayed a moderate Kerr nonlinearity, resulting in an extremely high nonlinear figure of merit as well as a nonlinear parameter that was high enough to support substantial parametric gain. Following the first report of micro-ring resonator (MRR) based frequency comb source driven by the Kerr optical nonlinearity in 2007 [88], the first fully integrated optical parametric oscillators were reported in $2010[76,77]$ that were also CMOS compatible. Since then the field of integrated micro-combs, or "Kerr combs" has become one of the largest fields in optics and photonics [87]. Integrated optical Kerr frequency comb sources, or "micro-combs" are a fundamentally new and powerful tool to accomplish many new functions on an integrated chip, due to their ability to provide highly coherent combs of multiple wavelength channels, at the same time offering a very high degree of wavelength spacing control. Optical micro-combs are produced via optical parametric oscillation driven by modulational instability gain in monolithic MRRs. They offer significant advantages over more traditional multi-wavelength sources. Many innovative applications have been reported based on integrated Kerr micro-combs, from filter-driven mode-locked lasers [89-92] to quantum optical photonic chips [93-101], ultrahigh bandwidth optical fiber data transmission [102-104], optical neural networks [105-107], integrated optical frequency synthesizers [108]. We refer the reader to extensive reviews of micro-combs have been presented recently [87, $109-116]$. The success of the new CMOS platforms silicon nitride and Hydex motivated the search for even higher performing CMOS compatible platforms such as amorphous silicon [117] and silicon rich silicon nitride [118] that display a combination of low linear loss, high nonlinearity and very low nonlinear loss.

All-optical signal processing based on nonlinear optics has attracted significant interest over the years for its ability to achieve ultrahigh bandwidth without needing optical to electronic (or visa versa) conversion. It has been particularly important for a wide range of signal processing functions for both telecommunications and RF/microwave applications. RF photonic applications range from radar systems to signal generation and processing, [119-168] and are attractive because of the ultra-high bandwidths that can be achieved as well as low transmission loss and strong electromagnetic interference immunity. There is a very wide range photonic RF approaches including techniques that map the optical filter response in the optical domain onto the RF domain. This is perhaps best represented by integrated devices based on stimulated Brillouin scattering [128-135], which has resulted in extremely high performance for RF resolution - being able to resolve features as fine as $32 \mathrm{MHz}$, at the same time 
achieving a stopband rejection $>55 \mathrm{~dB}$. A key approach to achieving reconfigurable transfer functions for adaptive signal processing has been based on the transversal filter method [136-140] that operate by generating weighted, progressively delayed replicas of an RF signal imprinted on an optical carrier and then summing them via photo-detection. Transverse filters can realize a wide range of RF signal processing functions by only adjusting the tap weights, and so this method is very attractive to realize advanced RF filters that need to be dynamically adaptive. Both discrete laser diode arrays $[141,142]$ as well as integrated and fibre Bragg grating arrays and sampled gratings [143] have been successful at supplying the required taps. However, while offering many advantages, these approaches suffer from significantly increased complexity as well as reduced performance due to limitations in the available number of taps. Alternative approaches, such as employing optical frequency comb sources achieved by electro-optic (EO) or acousto-optic (AO) modulation [142, 144, 145], can help overcome this problem, but they require multiple high frequency modulators that rely on high-frequency RF sources. Of these approaches, EO combs have been used the longest and have achieved significant success at realizing many powerful functions.

Integrated optical Kerr micro-combs are a relatively recent innovation and have been very successful offering many advantages over other approaches that use multi-wavelength sources for RF applications. They have been demonstrated to be capable of achieving extremely high bit rate telecommunications systems as well as a very wide range of RF signal processing functions [147-168]. They can generate frequency combs with a much larger spacing than electro-optic combs. EO combs and micro-combs are in fact complementary in many ways - EO combs focuses on finer wavelength spacings from 10's of $\mathrm{MHz}$ to $10-20 \mathrm{GHz}$, while integrated micro-combs typically have much wider spacings from 10's to 100's of $\mathrm{GHz}$ and even into the $\mathrm{THz}$ regime. Larger comb spacings yield much wider Nyquist zones that are needed to achieve large RF bandwidths, whereas finer spacings provide much larger numbers of wavelengths or RF "taps", but at the cost of a much smaller Nyquist zone, or RF bandwidth. Micro-combs can provide higher numbers of wavelengths together with still being able to provide a large FSR, in a compact footprint. For RF transversal filters the number of taps, or wavelengths, dictates the available number of channels for, for example, RF true time delays as well as determining the performance of RF filters $[125,161]$. Other systems such as beamforming devices [152] can also be greatly improved in terms of their quality factor and angular resolution. Other innovative approaches to filtering include techniques such as RF bandwidth scaling [165] that provide a certain bandwidth for each wavelength channel, and so the total operation bandwidth (maximum RF signal bandwidth that can be processed) will depend on the number of wavelengths, and will therefore be significantly enhanced by the use of micro-combs.

We have reported [147-168] a wide range of microwave and RF signal processing based on transversal filtering and bandwidth scaling methods based on Kerr micro-combs applied to RF and microwave spectral filters. We have achieved both integral and fractional order Hilbert transformers and differentiators, as well as RF integrators. We have examined the trade-offs involved between using wide spaced micro-combs with an FSR of 200GHz [149-151] with record low FSR spaced micro-combs at 49 $\mathrm{GHz}$, based on soliton crystals [162-179]. While $200 \mathrm{GHz}$ Kerr micro-combs have proven to be a powerful 
source for RF transversal filters, enabling high versatility as well as dynamic reconfigurability, the relatively large comb spacing FSR of $\sim 1.6 \mathrm{~nm}$ restricts the number of taps to typically less than 21 wavelengths within the $30 \mathrm{~nm}$ wide $\mathrm{C}$-band. This is important since transversal filters require optical amplifiers and spectral shapers that are typically only available in the telecommunications bands (1530$1620 \mathrm{~nm})$. This limitation in the tap number has restricted the performance of micro-comb based transversal RF filters in frequency selectivity, bandwidth, and dynamic versatility. To improve on this, we reported micro-comb-based photonic RF transversal filters operating with a record high number of taps up to 80 wavelengths over the C-band [157]. This is the highest number reported for micro-comb RF transversal filters, and has been enabled by a record low $49 \mathrm{GHz}$ FSR integrated Kerr micro-comb source. For filter applications [161] this enabled QRF factors for RF bandpass filters 4 times higher than that achievable with $200 \mathrm{GHz}$ combs. For temporal signal processing, this yields dramatic improvements in both bandwidth and reconfigurability. This photonics platform has been highly successful at a wide range of linear and nonlinear optical devices [77-92, 180-183] as well as quantum optical chips [93$101,184-191]$. Our results confirm the feasibility of achieving high performance advanced as well as adaptive RF transversal filters for use in high bandwidth RF signal processing systems, at the same time providing reduced cost, footprint, and complexity. Note that many of the functions that we implement with fiber components, such as the delay lines, can equally well be implemented with compact tunable dispersion compensators [192-195] as well as integrated detectors and modulators [196].

\section{Conclusion}

Here, we propose and demonstrate a reconfigurable microwave photonic intensity differentiator based on an integrated Kerr comb source. The Kerr optical comb was achieved via a CMOS-compatible nonlinear MRR, which greatly increased the processing bandwidth and has the potential for a low cost, size, and complexity processing system. By programming and shaping the individual comb lines' power according to the calculated tap weights, we successfully demonstrate the first, second, and third-order intensity differentiation of the RF signal. The RF amplitude and phase responses of the proposed differentiator are characterized, and system demonstrations of real-time differentiations are performed for Gaussian input pulses. We achieve good agreement between theory and experimental, thus verifying the effectiveness of our approach. In particular, our scheme provides a new way to implement microwave photonic intensity differentiators with high processing bandwidth and re-configurability, thus holding great promise for future ultra-high-speed computing and information processing.

\section{Declarations}

\section{Competing interests:}

The authors declare no competing interests.

\section{References}


1. S. T. Cundiff and A. M. Weiner, Nat. Photon. 4 (11), 760 (2010).

2. Z. Jiang, C. B. Huang, D. E. Leaird, and A. M. Weiner, Nat. Photon. 1 (8), 463 (2007).

3. M. Ferrera, Y. Park, L. Razzari, B. E. Little, S. T. Chu, R. Morandotti, D. J. Moss, and J. Azaña, Opt. Exp. 19 (23), 23153 (2011).

4. M. Ferrera, C. Reimer, A. Pasquazi, M. Peccianti, M. Clerici, L. Caspani, S. T. Chu, B. E. Little, R. Morandotti, and D. J. Moss, Opt. Exp. 22 (18), 21488 (2014).

5. J. Wu, J. Peng, B. Liu, T. Pan, H. Zhou, J.Mao, Y. Yang, C. Qiu, and Y. Su, Opt. Commun. 373, 44 (2016).

6. C. Monat, C. Grillet, M. Collins, A. Clark, J. Schroeder, C. Xiong, J. Li, L. O'Faolain, T. F. Krauss, B. J. Eggleton, and D. J. Moss, Nat. Commun. 5, 3246 (2014).

7. N. Q. Ngo, S. Yu, S. C. Tjin, and C. H. Kam, Opt. Commun. 230 (1-3), 115 (2004).

8. F. Li, Y. Park, and J. Azaña, J. Lightwave Technol. 27 (21), 4623 (2009).

9. Y. Han, Z. Li, and J. Yao, J. Lightwave Technol. 29 (22), 3470 (2011).

10. R. Ashrafi and J. Azaña, Opt. Exp. 20 (3), 2626 (2012).

11. Y. Yu, F. Jiang, H. Tang, L. Xu, X. Liu, J. Dong, and X. Zhang, Opt. Exp. 24 (11), 1739 (2016).

12. R. Slavik, Y. Park, M. Kulishov, R. Morandotti, and J. Azaña, Opt. Exp. 14 (22), 10699 (2006).

13. F. Liu, T. Wang, L. Qiang, T. Ye, Z. Zhang, M. Qiu, and Y. Su, Opt. Exp. 16 (20), 15880 (2008).

14. L. Zhang, J. Wu, X. Yin, X. Sun, P. Cao, X. Jiang, and Y. Su, IEEE Photon. J. 6 (2), 1 (2014).

15. T. Yang, J. Dong, L. Liu, S. Liao, S. Tan, L. Shi, D. Gao, and X. Zhang, Scientific Reports 4, 3960 (2014).

16. W. Liu, M. Li, R. S. Guzzon, E. J. Norberg, J. S. Parker, M. Lu, L. A. Coldren, and J. Yao, Nat. Photon. 10 (3), 190 (2016).

17. H. J. A. da Silva and J. J. O’Reilly, Opt. Lett. 14 (10), 526-528 (1989).

18. M. H. Asghari and J. Azaña, Opt. Commun. 281 (18), 4581-4588 (2008).

19. M. Stratmann, T. Pagel, and F. Mitschke, Phys. Rev. Lett. 95 (14), 143902 (2005).

20. F. Zeng and J. Yao, Photon. Technol. Lett. 18 (17-20), 2062 (2006).

21. S. Pan and J. Yao, Opt. Lett. 34 (9), 1312 (2009).

22. X. Li, J. Dong, Y. Yu, and X. Zhang, IEEE Photon. J. 23 (5), 308 (2011).

23. J. Xu, X. Zhang, J. Dong, D. Liu, and D. Huang, Opt. Lett. 32 (13), 1872 (2007).

24. P. Velanas, A. Bogris, A. Argyris, and D. Syvridis, J. Lightwave Technol. 26 (17-20), 3269 (2008).

25. M. Bolea, J. Mora, B. Ortega, and J. Capmany, Opt. Exp. 17 (7), 5023 (2009).

26. F. Ferdous, H. Miao, D. E. Leaird, K. Srinivasan, J. Wang, L. Chen, L. T. Varghese, and A. M. Weiner, Nat. Photon. 5 (12), 770 (2011).

27. M. H. Khan, H. Shen, Y. Xuan, L. Zhao, S. Xiao, D. E. Leaird, A. M. Weiner, and M. Qi, Nat. Photon. 4 (2), 117 (2010). 
28. A. V. Oppenheim, A. S. Willsky, S. H. Nawab, Signals \& systems (2nd ed.). (Prentice-Hall, Inc., 1996), p.957.

29. J. Capmany, J. Mora, I. Gasulla, J. Sancho, J. Lloret, and S. Sales, J. Lightwave Technol. 31 (4), 571 (2013).

30. H. Emami, N. Sarkhosh, L. A. Bui, and A. Mitchell, Opt. Lett. 33 (2), 98 (2008).

31. S. Mansoori and A. Mitchell, Photon. Technol. Lett. 16 (3), 879 (2004).

32. J. McClellan, T. W. Parks, and L. Rabiner, IEEE Transactions on Audio and Electroacoustics 21 (6), 506 (1973).

33. D. J. Moss, R. Morandotti, A. L. Gaeta, and M. Lipson, Nat. Photon. 7 (8), 597 (2013).

34. L. Razzari, D. Duchesne, M. Ferrera, R. Morandotti, S. Chu, B. E. Little, and D. J. Moss, Nat. Photon. 4 (1), 41 (2010).

35. T. G. Nguyen, M. Shoeiby, S. T. Chu, B. E. Little, R. Morandotti, A. Mitchell, and D. J. Moss, Opt. Exp. 23 (17), 22087 (2015).

36. J. Wu, P. Cao, X. Hu, X. Jiang, T. Pan, Y. Yang, C. Qiu, C. Tremblay, and Y. Su, Opt. Exp. 22, 26254 (2014).

37. A. Ortigosa-Blanch, J. Mora, J. Capmany, B. Ortega, and D. Pastor, Opt. Lett. 31 (6), 709 (2006).

38. E. Hamidi, D. E. Leaird, and A. M. Weiner, IEEE T. Microw. Theory 58 (11), 3269 (2010).

39. T. Herr, K. Hartinger, J. Riemensberger, C. Wang, E. Gavartin, R. Holzwarth, M. L. Gorodetsky, and T. J. Kippenberg, Nat. Photon. 6 (7), 480 (2012).

40. D.Duchesne, M.Ferrera, L.Razzari, R.Morandotti, B.Little, S.Chu, and D. J. Moss, "Nonlinear optics in ultra low loss high-index glass waveguide", Ch.14, pp269-294, in Frontiers in Guided Wave Optics and Optoelectronics, Edited by B. Pal, IN-TECH, Vienna, Austria, ISBN 978-953-7619-82-4 (2010).

41. S. Radic, and D. J. Moss, and B. J. Eggleton, "Nonlinear optics in communications: From crippling impairment to ultrafast tools", Chapter 20, p759-828 in Optical Fiber Telecommunications V: Components and Sub-systems, Ed. Ivan P. Kaminow, Tingye Li, and Alan E. Willner, Academic Press, Oxford, UK, February 2008.

42. J. Leuthold, C. Koos, and W. Freude, "Nonlinear silicon photonics," Nat. Photon., vol. 4, no. 8, pp. 535$544,2010$.

43. L. Li, P. G. Patki, Y.B. Kwon, et al. "All-optical regenerator of multi-channel signals," Nature Communications, vol. 8, Article: 884, 2017.

44. F.Li, et al.,"All-optical XOR logic gate for $40 \mathrm{~Gb} / \mathrm{s}$ DPSK signals via FWM in a Si nanowire," Opt. Express, vol.19, no.21, pp.20364-20371, 2011.

45. F. Li, et al., "Error-free All-Optical Demultiplexing at $160 \mathrm{~Gb} / \mathrm{s}$ via FWM in a Si Nanowire," Optics Express, vol. 18, no. 4, pp. 3905-3910, 2010.

46. H. Ji, et al., "1.28-Tb/s Demultiplexing of an OTDM DPSK Data Signal Using a Silicon Waveguide," Photonics Technology Letters, vol. 22, no. 23, pp. 1762-1764, 2010. 
47. C. Monat, et al., "Investigation of phase matching for third-harmonic generation in silicon slow light photonic crystal waveguides using Fourier optics," Optics Express, vol. 18, no. 7, pp. 6831-6840, 2010. DOI: 10.1364/OE.18.006831.

48. B. Corcoran, et al., "Optical Signal Processing on a Si Chip at $640 \mathrm{~Gb} / \mathrm{s}$ Using Slow-Light," Optics Express, vol. 18, no. 8, pp. 7770-7781, 2010.

49. V. G. Ta'eed, et al., "Integrated all-optical pulse regenerator in chalcogenide waveguides," Optics Letters, vol. 30, no. 21, pp. 2900-2902, 2005 . DOI: 10.1364/OL.30.002900.

50. M. Rochette, et al., "Bit-error-ratio improvement with 2R optical regenerators," IEEE Phot. Technology Lett., vol. 17, no. 4, pp. 908-910, 2005.

51. M. Ferrera, et al., "CMOS compatible integrated all-optical radio frequency spectrum analyzer," Optics Express, vol. 22, no. 18, pp. 21488 - 21498, 2014. DOI: 10.1364/OE.22.021488.

52. C. Monat, et al., "Integrated optical auto-correlator based on third-harmonic generation in a silicon photonic crystal waveguide," Nature Communications, vol. 5, Article:3246, 2014. doi:10.1038/ncomms4246.

53. F. Li, et al., "All-optical wavelength conversion for $10 \mathrm{~Gb} / \mathrm{s}$ DPSK signals in a silicon ring resonator," Optics Express, vol. 19, no. 23, pp. 22410-22416, 2011.

54. T. D. Vo, et al., "Silicon-Chip-Based Real-Time Dispersion Monitoring for 640 Gbit/s DPSK Signals," IEEE Journal of Lightwave Technology, vol. 29, no. 12, pp. 1790-1796, 2011.

55. M. Ferrera, et al., "All-optical 1st and 2nd order integration on a chip," Optics Express, vol. 19, no. 23, pp. 23153-23161, 2011.

56. B. Corcoran, et al., "Silicon nanowire based radio-frequency spectrum analyzer," Optics Express, vol. 18, no. 19, pp. 20190-20200, 2010.

57. B. Corcoran, et al., "Green light emission in silicon through slow-light enhanced third-harmonic generation in photonic-crystal waveguides," Nature Photonics, vol. 3, no. 4, pp. 206-210, 2009. doi:10.1038/nphoton.2009.28.

58. D. J. Moss, H. M. van Driel, and J. E. Sipe, "Dispersion in the anisotropy of optical third-harmonic generation in silicon," Opt. Lett., vol. 14, no. 1, pp. 57-59, 1989.

59. J. E. Sipe, D. J. Moss, and H. M. van Driel, "Phenomenological Theory of Optical Second- And ThirdHarmonic Generation Form Cubic Centrosymmetric Crystals," Phys. Rev. B, vol. 35, no. 3, pp. 11291141, 1987.

60. D. J. Moss, E. Ghahramani, J. E. Sipe, and H. M. van Driel, "Band-structure calculation of dispersion and anisotropy in $\chi \rightarrow(3)$ for third-harmonic generation in $\mathrm{Si}$, Ge, and GaAs," Phys. Rev. B, vol. 41, no. 3, pp. 1542-1560, 1990.

61. D. J. Moss, H. M. van Driel, and J. E. Sipe, "Third harmonic generation as a structural diagnostic of ion implanted amorphous and crystalline silicon," Appl. Phy. Lett., vol. 48, no. 17, pp. 1150, 1986.

62. D. J. Moss, et al., "Ultrafast all-optical modulation via two-photon absorption in silicon-insulator waveguides," Electronics Letters, vol. 41, no. 6, pp. 320-321, 2005. DOI:10.1049/el:20058051 
63. M. R. E. Lamont, et al.,"Two-photon absorption effects on self-phase-modulation-based 2R optical regeneration," Photonics Technology Letters, vol. 18, no. 10, pp. 1185-1187, 2006. DOI:10.1109/LPT.2006.874718.

64. A.Tuniz, G. Brawley, D. J. Moss, and B. J. Eggleton, "Two-photon absorption effects on Raman gain in single mode As2Se3 chalcogenide glass fiber," Optics Express, vol. 16, no. 22, pp. 18524-18534, 2008. DOI: 10.1364/OE.16.018524.

65. T.Monro, D.J.Moss, M. Bazylenko, C. Martijn de Sterke, and L. Poladian, “Observation of self-trapping of light in a self written channel in photosensitive glass", Physical Review Letters 804072 (1998).

66. M. Lee, et al., "Photosensitive post tuning of chalcogenide photonic crystal waveguides," Optics Express, vol. 15, no. 3, pp. 1277-1285, 2007.

67. S. Tomljenovic-Hanic, M. J. Steel, C. M. d. Sterke and D. J. Moss, "High-Q cavities in photosensitive photonic crystals", Optics Letters, 32, no. 5, pp. 542-544, 2007.

68. C.Grillet, et al., "Nanowire coupling to photonic crystal nanocavities for single photon sources," Opt.Express, vol.15, no.3, pp.1267-1276, 2007.

69. V. G. Ta'eed, et al., "Ultrafast all-optical chalcogenide glass photonic circuits”, Opt. Express, vol. 15, no. 15, pp.9205, 2007.

70. D. Freeman, et al., "Chalcogenide Glass Photonic Crystal Devices", Photonic and Electromagnetic Crystal Structures, Photonics and Nanostructures-Fundamentals and Applications, Science Direct Elsevier Publishing, vol. 6, no. 1, pp. 3-11, 2008.

71. C. Grillet, et al., "Characterization and modeling of Fano resonances in chalcogenide photonic crystal membranes", Optics Express, vol. 14, no. 1, pp. 369-376, 2006.

72. V. G. Ta'eed, et al., "Self-phase modulation based integrated optical regeneration in chalcogenide waveguides", IEEE Journal of Selected Topics in Quantum Electronics, vol. 12, no. 3, pp. 360-370, 2006.

73. M. Shokooh-Saremi, et al., "High performance Bragg gratings in chalcogenide rib waveguides written with a modified Sagnac interferometer: experiment and modeling", Journal of the Optical Society of America B, vol. 23, no. 7, pp. 1323-1331, 2006.

74. M. R. E. Lamont, et al., "Error-free wavelength conversion via cross phase modulation in $5 \mathrm{~cm}$ of As2S3 chalcogenide glass rib waveguide", Electronics Letters, vol. 43, pp. 945-947, 2007.

75. K. Ikeda, R. E. Saperstein, N. Alic, and Y. Fainman, "Thermal and Kerr nonlinear properties of plasmadeposited silicon nitride/silicon dioxide waveguides", Opt. Express, vol. 16, pp. 12987-12994, 2008.

76. J.S. Levy et al., "CMOS-compatible multiple-wavelength oscillator for on-chip optical interconnects," Nature Phot. vol. 4 (1) pp. 37-40, 2010.

77. L. Razzari, et al., "CMOS-compatible integrated optical hyper-parametric oscillator," Nature Photonics, vol. 4, no. 1, pp. 41-45, 2010.

78. D. J. Moss, R. Morandotti, A. L. Gaeta, et al., "New CMOS-compatible platforms based on silicon nitride and Hydex for nonlinear optics," Nature Photonics, vol. 7, no. 8, pp. 597-607, 2013. 
79. M. Ferrera, L. Razzari, D. Duchesne, et al., "Low-power continuous-wave nonlinear optics in doped silica glass integrated waveguide structures," Nature Photonics, vol. 2, no. 12, pp. 737-740, 2008.

80. A. Pasquazi, M. Peccianti, Y. Park, B. E. Little, S. T. Chu, R. Morandotti, J. Azaña, and D. J. Moss, “Subpicosecond phase-sensitive optical pulse characterization on a chip”, Nature Photonics, vol. 5, no. 10, pp. 618 - 623 (2011).

81. D. Duchesne, M. Peccianti, M. R. E. Lamont, et al., "Supercontinuum generation in a high index doped silica glass spiral waveguide," Optics Express, vol. 18, no, 2, pp. 923-930, 2010.

82. M. Ferrera, et al., “On-chip CMOS-compatible all-optical integrator”, Nature Communications, vol. 1, Article 29, 2010.

83. A. Pasquazi, et al., "All-optical wavelength conversion in an integrated ring resonator," Optics Express, vol. 18, no. 4, pp. 3858-3863, 2010.

84. A. Pasquazi, Y. Park, J. Azana, et al., "Efficient wavelength conversion and net parametric gain via Four Wave Mixing in a high index doped silica waveguide," Optics Express, vol. 18, no. 8, pp. 76347641, 2010.

85. M. Peccianti, M. Ferrera, L. Razzari, et al., "Subpicosecond optical pulse compression via an integrated nonlinear chirper," Optics Express, vol. 18, no. 8, pp. 7625-7633, 2010.

86. D. Duchesne, M. Ferrera, L. Razzari, et al., “Efficient self-phase modulation in low loss, high index doped silica glass integrated waveguides," Optics Express, vol. 17, no. 3, pp. 1865-1870, 2009.

87. A. Pasquazi, M. Peccianti, L. Razzari, D. J. Moss, S. Coen, M. Erkintalo, Y. K. Chembo, T. Hansson, S. Wabnitz, P. Del'Haye, X. X. Xue, A. M. Weiner, and R. Morandotti, “Micro-combs: A novel generation of optical sources," Physics Reports, vol. 729, pp. 1-81, Jan 27. 2018.

88. P. Del'Haye, A. Schliesser, O. Arcizet, T. Wilken, R. Holzwarth, and T. J. Kippenberg, “Optical frequency comb generation from a monolithic microresonator”, Nature, vol. 450, pp. 1214-1217, 2007.

89. M. Peccianti, et al., "Demonstration of an ultrafast nonlinear microcavity modelocked laser”, Nature Communications, vol. 3, pp. 765, 2012.

90. M. Kues, et al., "Passively modelocked laser with an ultra-narrow spectral width”, Nature Photonics, vol. 11, no. 3, pp. 159, 2017.

91. A. Pasquazi, L. Caspani, M. Peccianti, et al., "Self-locked optical parametric oscillation in a CMOS compatible microring resonator: a route to robust optical frequency comb generation on a chip," Optics Express, vol. 21, no. 11, pp. 13333-13341, 2013.

92. A. Pasquazi, M. Peccianti, B. E. Little, et al., "Stable, dual mode, high repetition rate mode-locked laser based on a microring resonator," Optics Express, vol. 20, no. 24, pp. 27355-27362, 2012.

93. C. Reimer, et al., "Integrated frequency comb source of heralded single photons," Optics Express, vol. 22, no. 6, pp. 6535-6546, 2014.

94. C. Reimer, et al., "Cross-polarized photon-pair generation and bi-chromatically pumped optical parametric oscillation on a chip", Nature Communications, vol. 6, Article 8236, 2015. DOI: $10.1038 /$ ncomms 9236 
95. L. Caspani, C. Reimer, M. Kues, et al., "Multifrequency sources of quantum correlated photon pairs on-chip: a path toward integrated Quantum Frequency Combs," Nanophotonics, vol. 5, no. 2, pp. 351362, 2016.

96. C. Reimer et al., "Generation of multiphoton entangled quantum states by means of integrated frequency combs," Science, vol. 351, no. 6278, pp. 1176-1180, 2016.

97. M. Kues, et al., "On-chip generation of high-dimensional entangled quantum states and their coherent control", Nature, vol. 546, no. 7660, pp. 622-626, 2017.

98. P. Roztocki, M. Kues, C. Reimer, B. Wetzel, S. Sciara, Y. Zhang, A. Cino, B. E. Little, S. T. Chu, D. J. Moss, and R. Morandotti, "Practical system for the generation of pulsed quantum frequency combs," Optics Express, vol. 25, no. 16, pp. 18940-18949, 2017.

99. Y. Zhang, et al., "Induced photon correlations through superposition of two four-wave mixing processes in integrated cavities", Laser and Photonics Reviews, vol. 14, no. 7, pp. 2000128, 2020. DOI: 10.1002/lpor.202000128

100. M. Kues, C. Reimer, A. Weiner, J. Lukens, W. Munro, D. J. Moss, and R. Morandotti, "Quantum Optical Micro-combs", Nature Photonics, vol. 13, no.3, pp. 170-179, 2019.

101. C. Reimer, et al.,"High-dimensional one-way quantum processing implemented on d-level cluster states", Nature Physics, vol. 15, no.2, pp. 148-153, 2019.

102. P. Marin-Palomo, et al., "Microresonator-based solitons for massively parallel coherent optical communications", Nature, vol.546, 274, 2017.

103. J. Pfeifle, V. Brasch, M. Lauermann, Y. Yu, D. Wegner, T. Herr, K. Hartinger, et al., "Coherent terabit communications with microresonator Kerr frequency combs", Nature Photonics, vol. 8, no. 5, pp. 375380, 2014.

104. B. Corcoran, et al., "Ultra-dense optical data transmission over standard fiber with a single chip source", Nature Communications, vol. 11, Article:2568, 2020.

105. X. Xu, et al., "Photonic perceptron based on a Kerr microcomb for scalable high speed optical neural networks", Laser and Photonics Reviews, vol. 14, no. 8, 2020. DOl:10.1002/Ipor.202000070.

106. X. Xu et al., "11 TOPs photonic convolutional accelerator for optical neural networks", Nature, vol. $589,44-51$ (2021).

107. J. Feldmann, et al., "Parallel convolution processing using an integrated photonic tensor core", arXiv:2002.00281, 2020.

108. D. T. Spencer, et al., "An optical-frequency synthesizer using integrated photonics", Nature, vol. 557, no. 7703, pp. 81-85, 2018.

109. T.Kippenberg, A.Gaeta, M.Lipson, and M.Gorodetsky, "Dissipative Kerr solitons in optical microresonators," Science, vol. 361, no. 6402, 2018.

110. A. L. Gaeta, M. Lipson, and T.J.Kippenberg, "Photonic-chip-based frequency combs," Nature Photonics, vol. 13, no. 3, pp. 158-169, Mar. 2019. 
111. P. Del'Haye, T. Herr, E. Gavartin, M. L. Gorodetsky, R. Holzwarth, and T. J. Kippenberg, "Octave spanning tunable frequency comb from a microresonator", Physical Review Letters, vol. 107, no. 6 , pp. 063901, 2011.

112. T. J. Kippenberg, R. Holzwarth, S.A.Diddams, "Microresonator-based optical frequency combs", Science, vol. 332, no. 6029, pp.555-559, 2011.

113. T. Herr, V. Brasch, J. D. Jost, C. Y. Wang, N. M. Kondratiev, M. L. Gorodetsky, et al., "Temporal solitons in optical microresonators", Nature Photonics, vol. 8, no. 2, pp. 145-152, 2014.

114. F. Ferdous, H. Miao, D. E. Leaird, K. Srinivasan, J. Wang, L. Chen, L. T. Varghese, and A. M. Weiner, "Spectral line-by-line pulse shaping of on-chip microresonator frequency combs", Nature Photonics, vol. 5, no. 12, pp. 770, 2011.

115. X. Xue, P. H. Wang, Y. Xuan, M. Qi, and A. M. Weiner, "Microresonator Kerr frequency combs with high conversion efficiency", Laser \& Photonics Reviews, vol. 11, no. 1, 2017.

116. X. Xue, M. Qi, and A. M. Weiner, “Normal-dispersion microresonator Kerr frequency combs”, Nanophotonics, vol. 5, no. 2, pp. 244-262, 2016.

117. C. Grillet, et al., "Amorphous Silicon Nanowires with Record High Nonlinearity, FOM, and Optical Stability", Optics Express, vol. 20, no. 20. pp. 22609-22615, 2012.

118. J. W. Choi, B. Sohn, G. F. R. Chen, D. K. T. Ng, and D. T. H. Tan, "Soliton-effect optical pulse compression in CMOS-compatible ultra-silicon-rich nitride waveguides," APL Photonics, HIBSP2019, pp. 110804, 2020.

119. J. Capmany, and D. Novak, "Microwave photonics combines two worlds," Nat. Photonics, vol. 1, no. 6, pp. 319-330, 2007.

120. J. P. Yao, "Microwave photonics," Journal of Lightwave Technol., vol. 27, no. 1-4, pp. 314-335, JanFeb. 2009.

121. D. Marpaung, J. Yao, and J. Capmany, "Integrated microwave photonics," Nature Photonics, vol. 13, no. 2, 80-90, Feb. 2019.

122. J.Azaña, "Ultrafast analog all-optical signal processors based on fiber-grating devices," IEEE Photonics Journal, vol. 2, no. 3, pp.359-386. 2010.

123. J. Capmany, B. Ortega, and D. Pastor, "A tutorial on microwave photonic filters," J. Lightwave Technol., vol. 24, no. 1, pp. 201-229, 2006.

124. V. R. Supradeepa et al., "Comb-based radiofrequency photonic filters with rapid tunability and high selectivity," Nat. Photonics, vol. 6, no. 3, pp. 186-194, Mar.2012.

125. J. Wu, X. Xu, T. G. Nguyen, S. T. Chu, B. E. Little, R. Morandotti, A. Mitchell, and D. J. Moss, "RF Photonics: An Optical Microcombs' Perspective," IEEE J. Sel. Top. Quantum Electron., vol. 24, no. 4, pp. 6101020, Jul-Aug. 2018.

126. V. Torres-Company, and A. M. Weiner, "Optical frequency comb technology for ultra-broadband radiofrequency photonics," Laser Photonics Rev, vol. 8, no. 3, pp. 368-393, May. 2014. 
127. Z. Jiang, C. B. Huang, D. E. Leaird, and A. M. Weiner, "Optical arbitrary waveform processing of more than 100 spectral comb lines," Nat Photonics, vol. 1, no. 8, pp. 463-467, Aug. 2007.

128. Y. Liu, J. Hotten, A. Choudhary, B. J. Eggleton, and D. Marpaung, "All-optimized integrated RF photonic notch filter," Opt. Lett., vol. 42, no. 22, pp. 4631-4634, Nov 15. 2017.

129. Y. Liu, D. Marpaung, A. Choudhary, J. Hotten, and B. J. Eggleton, "Link performance optimization of chip-based Si3N4 microwave photonic filters," J. Lightwave Technol., vol. 36, no. 19, pp. 4361-4370, 2018.

130. Y. Liu, Y. Yu, S. X. Yuan, X. B. Xu, and X. L. Zhang, "Tunable megahertz bandwidth microwave photonic notch filter based on a silica microsphere cavity," Opt. Lett., vol. 41, no. 21, pp. 5078-5081, Nov 1. 2016.

131. D. Marpaung et al., "Low-power, chip-based stimulated Brillouin scattering microwave photonic filter with ultrahigh selectivity," Optica, vol. 2, no. 2, pp. 76-83, Feb 20. 2015.

132. A. Choudhary et al., "Advanced integrated microwave signal processing with giant on-chip Brillouin gain," J. Lightwave Technol., vol. 35, no. 4, pp. 846-854, Feb 15. 2017.

133. D. Marpaung, B. Morrison, R. Pant, and B. J. Eggleton, "Frequency agile microwave photonic notch filter with anomalously high stopband rejection," Opt. Lett., vol. 38, no. 21, pp. 4300-4303, Nov 1. 2013.

134. X. Q. Zhu, F. Y. Chen, H. F. Peng, and Z. Y. Chen, “Novel programmable microwave photonic filter with arbitrary filtering shape and linear phase," Opt. Express, vol. 25, no. 8, pp. 9232-9243, Apr 17. 2017.

135. F. Jiang, Y. Yu, H. T. Tang, L. Xu, and X. L. Zhang, "Tunable bandpass microwave photonic filter with ultrahigh stopband attenuation and skirt selectivity," Opt. Express, vol. 24, no. 16, pp. 18655-18663, Aug 8. 2016.

136. Z. J. Zhu, H. Chi, T. Jin, S. L. Zheng, X. F. Jin, and X. M. Zhang, "All-positive-coefficient microwave photonic filter with rectangular response," Opt. Lett., vol. 42, no. 15, pp. 3012-3015, Aug 1. 2017.

137. G. Yu, W. Zhang, and J. A. R. Williams, "High-performance microwave transversal filter using fiber Bragg grating arrays," IEEE Photonic Tech L, vol. 12, no. 9, pp. 1183-1185, Sep. 2000.

138. J. S. Leng, W. Zhang, and J. A. R. Williams, "Optimization of superstructured fiber Bragg gratings for microwave photonic filters response," IEEE Photonic Tech L, vol. 16, no. 7, pp. 1736-1738, Jul. 2004.

139. D. B. Hunter, R. A. Minasian, and P. A. Krug, "Tunable optical transversal filter based on chirped gratings," Electron. Lett., vol. 31, no. 25, pp.2205-2207, Dec 7. 1995.

140. E. Hamidi, D. E. Leaird, and A. M. Weiner, "Tunable Programmable Microwave Photonic Filters Based on an Optical Frequency Comb," IEEE Journal of Microwave Theory, vol. 58, no. 11, pp. 3269-3278, Nov. 2010.

141. R. Wu, V. R. Supradeepa, C. M. Long, D. E. Leaird, and A. M. Weiner, "Generation of very flat optical frequency combs from CW lasers using cascaded intensity and phase modulators driven by tailored RF waveforms," Opt. Lett., vol.35, no.19, 3234. 2010. 
142. S. Mansoori, and A.Mitchell, "RF transversal filter using an AOTF," IEEE Photonic Tech L, vol. 16, no. 3 , pp. 879-881, 2004.

143. M. Delgado-Pinar, J. Mora, A. Diez, M. V. Andres, B. Ortega, and J. Capmany, "Tunable and reconfigurable microwave filter by use of a Bragg-grating-based acousto-optic superlattice modulator," Opt. Lett., vol. 30, no. 1, pp. 8-10, Jan 1. 2005.

144. W. Z. Li, and J. P. Yao, "Optical frequency comb generation based on repeated frequency shifting using two Mach-Zehnder modulators and an asymmetric Mach-Zehnder interferometer," Opt. Express, vol. 17, no. 26, pp. 23712-23718, Dec 21. 2009.

145. C. H. Chen, C. He, D. Zhu, R. H. Guo, F. Z. Zhang, and S. L. Pan, "Generation of a flat optical frequency comb based on a cascaded polarization modulator and phase modulator," Opt. Lett., vol. 38, no. 16, pp. 3137-3139, Aug 15. 2013.

146. T. Saitoh, M. Kourogi, and M. Ohtsu, "An optical frequency synthesizer using a waveguide-type optical frequency comb generator at 1.5-mu m wavelength," IEEE Photonic Tech L, vol. 8, no. 11, pp. 1543-1545, Nov. 1996.

147. T. G. Nguyen et al., "Integrated frequency comb source-based Hilbert transformer for wideband microwave photonic phase analysis," Opt. Express, vol. 23, no. 17, pp. 22087-22097, Aug. 2015.

148. X. Xue, et al., "Programmable single-bandpass photonic RF filter based on a Kerr comb from a microring," Journal of Lightwave Technol., vol. 32, no. 20, pp. 3557-3565, Oct. 2014.

149. X. Xu et al., "Reconfigurable broadband microwave photonic intensity differentiator based on an integrated optical frequency comb source," APL Photonics, vol. 2, no. 9, 096104, Sep. 2017.

150. X. Xu, M. Tan, J. Wu, R. Morandotti, A. Mitchell, and D. J. Moss, "Microcomb-based photonic RF signal processing”, IEEE Photonics Technology Letters, vol. 31 no. 23 1854-1857, 2019.

151. X. Xu, et al., "Advanced RF and microwave functions based on an integrated optical frequency comb source," Opt. Express, vol. 26, no. 3, pp. 2569-2583, Feb. 2018.

152. X. Xue, et al., "Microcomb-based true-time-delay network for microwave beamforming with arbitrary beam pattern control," Journal of Lightwave Technology, vol. 36, no. 12, pp. 2312-2321, Jun. 2018.

153. X. Xu, et al., "Broadband RF channelizer based on an integrated optical frequency Kerr comb source," Journal of Lightwave Technology, vol. 36, no. 19, pp. 4519-4526, 2018.

154. X. Xu, et al., "Continuously tunable orthogonally polarized RF optical single sideband generator based on micro-ring resonators," Journal of Optics, vol. 20, no. 11, pp. 115701. 2018.

155. X. Xu, et al., "Orthogonally polarized RF optical single sideband generation and dual-channel equalization based on an integrated microring resonator," Journal of Lightwave Technology, vol. 36, no. 20, pp. 4808-4818. 2018.

156. X. Xu, et al., "Photonic microwave true time delays for phased array antennas using a $49 \mathrm{GHz}$ FSR integrated optical micro-comb source," Photonics Res, vol. 6, no. 5, pp. B30-B36, 2018.

157. X. Xu, et al., "Advanced adaptive photonic RF filters with 80 taps based on an integrated optical micro-comb source," Journal of Lightwave Technology, vol. 37, no. 4, pp. 1288-1295, 2019. 
158. X. Xu, et al., Broadband microwave frequency conversion based on an integrated optical micro-comb source", Journal of Lightwave Technology, vol. 38 no. 2, pp. 332-338, 2020.

159. M. Tan, et al., "Photonic RF and microwave filters based on $49 \mathrm{GHz}$ and $200 \mathrm{GHz}$ Kerr microcombs", Optics Comm. vol. 465, 125563. 2020.

160. X. Xu, et al., "Broadband photonic RF channelizer with 90 channels based on a soliton crystal microcomb", Journal of Lightwave Technology, vol. 38, no. 18, 5116 - 5121, Sept. 15 (2020). doi: 10.1109/JLT.2020.2997699

161. X. Xu, et al., "Photonic RF and microwave integrator with soliton crystal microcombs", IEEE Transactions on Circuits and Systems II: Express Briefs, vol. 67, no. 12, pp. 3582-3586, 2020.

162. X.Xu, et al.,“Photonic RF phase-encoded signal generation with a microcomb source”, J.Lightwave Technology, vol.38 (7) pp.1722-1727, 2020.

163. M. Tan, et al., "Microwave and RF photonic fractional Hilbert transformer based on a $50 \mathrm{GHz}$ Kerr micro-comb", Journal of Lightwave Technology, vol. 37, no. 24, pp. 6097 - 6104, 2019.

164. M. Tan, et al., "RF and microwave fractional differentiator based on photonics", IEEE Transactions on Circuits and Systems: Express Briefs, vol. 67, no.11, pp. 2767-2771, 2020.

165. X. Xu, et al., "High performance RF filters via bandwidth scaling with Kerr micro-combs," APL Photonics, vol. 4, no. 2, pp. 026102. 2019.

166. M. Tan, et al., "Photonic RF arbitrary waveform generator based on a soliton crystal micro-comb source”, Journal of Lightwave Technology, vol. 38, no. 22, pp. 6221-6226, Oct 22, 2020.

167. J. Wu, X. Xu, T. G. Nguyen, S. T. Chu, B. E. Little, R. Morandotti, A. Mitchell, and D. J. Moss, "RF Photonics: An Optical Microcombs' Perspective," IEEE J. Sel. Top. Quantum Electron., vol. 24, no. 4, pp. 6101020, Jul-Aug. 2018.

168. Mengxi Tan, X. Xu, J. Wu, T. G. Nguyen, S. T. Chu, B. E. Little, R. Morandotti, A. Mitchell, and David J. Moss, "Photonic Radio Frequency Channelizers based on Kerr Optical Micro-combs", Journal of Semiconductors42 (4), 041302 (2021). (ISSN 1674-4926). DOI:10.1088/1674-4926/42/4/041302.

169. H.Bao, L.Olivieri, M.Rowley, S.T. Chu, B.E. Little, R.Morandotti, D.J. Moss, J.S.T. Gongora, M.Peccianti and A.Pasquazi, "Laser Cavity Solitons and Turing Patterns in Microresonator Filtered Lasers: properties and perspectives", Paper No. LA203-5, Paper No. 11672-5, SPIE LASE, SPIE Photonics West, San Francisco CA March 6-11 (2021). DOI:10.1117/12.2576645

170. Mengxi Tan, X. Xu, J. Wu, A. Boes, T. G. Nguyen, S. T. Chu, B. E. Little, R. Morandotti, A. Mitchell, and David J. Moss, "Advanced microwave signal generation and processing with soliton crystal microcombs", or "Photonic convolutional accelerator and neural network in the Tera-OPs regime based on Kerr microcombs", Paper No. 11689-38, PW210-0E201-67, Integrated Optics: Devices, Materials, and Technologies XXV, SPIE Photonics West, San Francisco CA March 6-11 (2021). DOI: $10.1117 / 12.2584017$

171. Mengxi Tan, Bill Corcoran, Xingyuan Xu, Andrew Boes, Jiayang Wu, Thach Nguyen, Sai T. Chu, Brent E. Little, Roberto Morandotti, Arnan Mitchell, and David J. Moss, "Optical data transmission at 40 Terabits/s with a Kerr soliton crystal microcomb", Paper No.11713-8, PW210-0E803-23, Next- 
Generation Optical Communication: Components, Sub-Systems, and Systems X, SPIE Photonics West, San Francisco CA March 6-11 (2021). DOI:10.1117/12.2584014

172. Mengxi Tan, X. Xu, J. Wu, A. Boes, T. G. Nguyen, S. T. Chu, B. E. Little, R. Morandotti, A. Mitchell, and David J. Moss, "RF and microwave photonic, fractional differentiation, integration, and Hilbert transforms based on Kerr micro-combs", Paper No. 11713-16, PW210-OE803-24, Next-Generation Optical Communication: Components, Sub-Systems, and Systems X, SPIE Photonics West, San Francisco CA March 6-11 (2021). DOI:10.1117/12.2584018

173. Mengxi Tan, X. Xu, J. Wu, A. Boes, T. G. Nguyen, S. T. Chu, B. E. Little, R. Morandotti, A. Mitchell, and David J. Moss, "Broadband photonic RF channelizer with 90 channels based on a soliton crystal microcomb", or "Photonic microwave and RF channelizers based on Kerr micro-combs", Paper No. 11685-22, PW210-OE106-49, Terahertz, RF, Millimeter, and Submillimeter-Wave Technology and Applications XIV, SPIE Photonics West, San Francisco CA March 6-11 (2021).

DOI:10.1117/12.2584015

174. X. Xu, M. Tan, J. Wu, S. T. Chu, B. E. Little, R. Morandotti, A. Mitchell, B. Corcoran, D. Hicks, and D. J. Moss, "Photonic perceptron based on a Kerr microcomb for scalable high speed optical neural networks", IEEE Topical Meeting on Microwave Photonics (MPW), pp. 220-224,.Matsue, Japan, November 24-26, 2020. Electronic ISBN:978-4-88552-331-

\section{DOI: $10.23919 / M W P 48676.2020 .9314409$}

175. Mengxi Tan, Bill Corcoran, Xingyuan Xu, Andrew Boes, Jiayang Wu, Thach Nguyen, S.T. Chu, B. E. Little, Roberto Morandotti, Arnan Mitchell, and David J. Moss, "Ultra-high bandwidth optical data transmission with a microcomb", IEEE Topical Meeting on Microwave Photonics (MPW), pp. 7882.Virtual Conf., Matsue, Japan, November 24-26, 2020. Electronic ISBN:978-4-88552-331-1.

DOI: 10.23919/MWP48676.2020.9314476

176. M. Tan, X. Xu, J. Wu, R. Morandotti, A. Mitchell, and D. J. Moss, "RF and microwave high bandwidth signal processing based on Kerr Micro-combs", Advances in Physics X, VOL. 6, NO. 1, 1838946 (2020). DOI:10.1080/23746149.2020.1838946.

177. Mengxi Tan, Xingyuan Xu, Jiayang Wu, Thach G. Nguyen, Sai T. Chu, Brent E. Little, Roberto Morandotti, Arnan Mitchell, and David J. Moss, "Photonic Radio Frequency Channelizers based on Kerr Micro-combs and Integrated Micro-ring Resonators", JOSarXiv.202010.0002.

178. Mengxi Tan, Xingyuan Xu, David Moss "Tunable Broadband RF Photonic Fractional Hilbert Transformer Based on a Soliton Crystal Microcomb", Preprints, DOI: 10.20944/preprints202104.0162.v1

179. Mengxi Tan, X. Xu, J. Wu, T. G. Nguyen, S. T. Chu, B. E. Little, R. Morandotti, A. Mitchell, and David J. Moss, "Orthogonally polarized Photonic Radio Frequency single sideband generation with integrated micro-ring resonators", Journal of Semiconductors42 (4), 041305 (2021).DOI: 10.1088/16744926/42/4/041305.

180. H. Bao, et al., "Laser cavity-soliton microcombs," Nature Photonics, vol. 13, no. 6, pp. 384-389, Jun. 2019. 
181. H. Bao et al., "Turing patterns in a fibre laser with a nested micro-resonator: robust and controllable micro-comb generation", Physical Review Research, vol. 2, pp. 023395, 2020.

182. L. D. Lauro, J. Li, D. J. Moss, R. Morandotti, S. T. Chu, M. Peccianti, and A. Pasquazi, "Parametric control of thermal self-pulsation in micro-cavities," Opt. Lett. vol. 42, no. 17, pp. 3407-3410, Aug. 2017.

183. H. Bao, et al., "Type-II micro-comb generation in a filter-driven four wave mixing laser," Photonics Research, vol. 6, no. 5, pp. B67-B73, 2018.

184. Stefania Sciara, Piotr Roztocki, Bennet Fisher, Christian Reimer, Luis Romero Cortez, William J. Munro, David J. Moss, Alfonso C. Cino, Lucia Caspani, Michael Kues, J. Azana, and Roberto Morandotti, "Scalable and effective multilevel entangled photon states: A promising tool to boost quantum technologies", Invited Review, Nanophotonics, vol. 10, pp. 1-17 (2021). DOI: 10.1515/nanoph-2021-0510.

185. Bennet Fischer, Mario Chemnitz, Benjamin Maclellan, Piotr Roztocki, Robin Helsten, Benjamin Wetzel, Brent E. Little, Sai T. Chu, David Moss, José Azaña, And Roberto Morandotti, "Autonomous on-chip interferometry for reconfigurable optical waveform generation", Optica 8, No. 9, 1 - 9 (2021). DOI:10.1364/OPTICA.435435.

186. Marco Piccardo et al., "Roadmap on multimode light shaping", IOP Journal of Optics, Vol. 23, 1$75(2021)$.

187. Fischer, P. Roztocki, C. Reimer, S. Sciara, Y. Zhang, M. Islam, L.R. Cortes, S. Bharadwaj, D.J. Moss, J. Azana, L. Caspani, M. Kues, R. Morandotti, Chaper 8, "Nonlinear and quantum effects in integrated microcavities", Nonlinear Meta-Optics, Edited by Costantino De Angelis, Giuseppe Leo, Dragomir N. Neshev, Taylor \& Francis (2020). CRC Press. DOI:10.1201/b22515.

188. Stefania Sciara, Piotr Roztocki, Cristina Rimoldi, Mario Chemnitz, Louis Romero Cortes, Michael Kues, David J. Moss, Lucia Caspani, José Azaña, and Roberto Morandotti, “Generation and Processing of Complex Photon States with Quantum Frequency Combs", IEEE Photonics Technology Letters, Vol. 31, Issue 23, 1862-1865 (2019). DOI: 10.1109/LPT.2019.2944564.

189. Stefania Sciara, Christian Reimer, Michael Kues, Piotr Roztocki, Alfonso Cino, David J. Moss, Lucia Caspani, William J. Munro, and Roberto Morandotti, "Universal N-Partite d-Level Pure-State Entanglement Witness Based on Realistic Measurement Settings", Physical Review Letters, Vol. 122, 120501 (2019). DOI: 10.1103/PhysRevLett.122.120501.

190. Christian Reimer, Yanbing Zhang, Piotr Roztocki, Stefania Sciara, Lucia Caspani, Luis Romero Cortés, Mehedi Islam, Bennet Fischer, Benjamin Wetzel, Alfonso Cino, Sai T Chu, Brent Little, David Moss, José Azaña, Michael Kues, Roberto Morandotti, "On-chip frequency combs and telecommunications signal processing meet quantum optics", Frontiers of Optoelectronics, Vol. 11 (2), 134-147 (2018). DOI: 10.1007/s12200-018-0814-0.

191. MacLellan, B., Roztocki, P., Kues, M., Reimer, C., Romero Cortés, L., Zhang, Y., Sciara, S., Wetzel, B., Cino, A., Chu, S.T., Little, B.E., Moss, D.J., Caspani, L., Azaña, J., Morandotti, R., “Generation and 
Coherent Control of Pulsed Quantum Frequency Combs", Journal of Visualized Experiments (JoVE), Vol. 136, e57517 (2018) doi:10.3791/57517; DOI:10.3791/5751.

192. D. J. Moss, M. Lamont, S. Mclaughlin, G. Randall, P. Colbourne, S. Kiran and C. A. Hulse,"Tunable dispersion and dispersion slope compensators for $10 \mathrm{~Gb} / \mathrm{s}$ using all-pass multicavity etalons", IEEE Photonics Technology Letters, vol. 15, pp. 730, 2003.

193. L. M. Lunardi, D. Moss, S. Chandrasekhar, L. L. Buhl, A. Hulse, P. Colbourne, G. Randall, S. Mclaughlin, "Tunable dispersion compensators based on multi-cavity all-pass etalons for $40 \mathrm{~Gb} / \mathrm{s}$ systems", IEEE J. Lightwave Technology, vol. 20, pp. 2136, 2002.

194. D. J. Moss, et al., "Multichannel tunable dispersion compensation using all-pass multicavity etalons", TuT2 Optical Fiber Communications Conf., Anaheim (2002). Technical Digest (IEEE Cat. No.02CH37339). Opt Soc. America. Part vol.1, 2002, pp. 132-3. Washington, DC, USA.

195. D. J. Moss, et al., "Tunable dispersion compensation at $10 \mathrm{~Gb} / \mathrm{s}$ and $40 \mathrm{~Gb} / \mathrm{s}$ using multicavity allpass etalons", Optical Fiber Communications Conf. TuD1, p162, Atlanta, GA, March (2003). Digest (IEEE Cat. No.03CH37403). OSA. Part vol.1, 2003, pp. 162-3. Washington, DC, USA.

196. T.Ido, H.Sano, D.J.Moss, S.Tanaka, and A.Takai, "Strained InGaAs/InAIAs MQW electroabsorption modulators with large bandwidth and low driving voltage", IEEE Photonics Technology Letters 61207 (1994). DOI: $10.1109 / 68.329640$.

\section{Table 1}

TABLE I. Tap coefficients for first-order, second-order, and third-order differentiation

\begin{tabular}{|lll|}
\hline Order of differentiation & Number of taps & Tap coefficients \\
\hline First-order & 8 & {$[-0.0226,0.0523,-0.1152,1,-1,0.1152,-0.052,0.0226]$} \\
\hline Second-order & 6 & {$[0.0241,-0.1107,0.0881,0.0881,-0.1107,0.0241]$} \\
\hline Third-order & 6 & {$[0.0450,-0.4076,1,-1,0.4076,-0.0450]$} \\
\hline
\end{tabular}

\section{Figures}



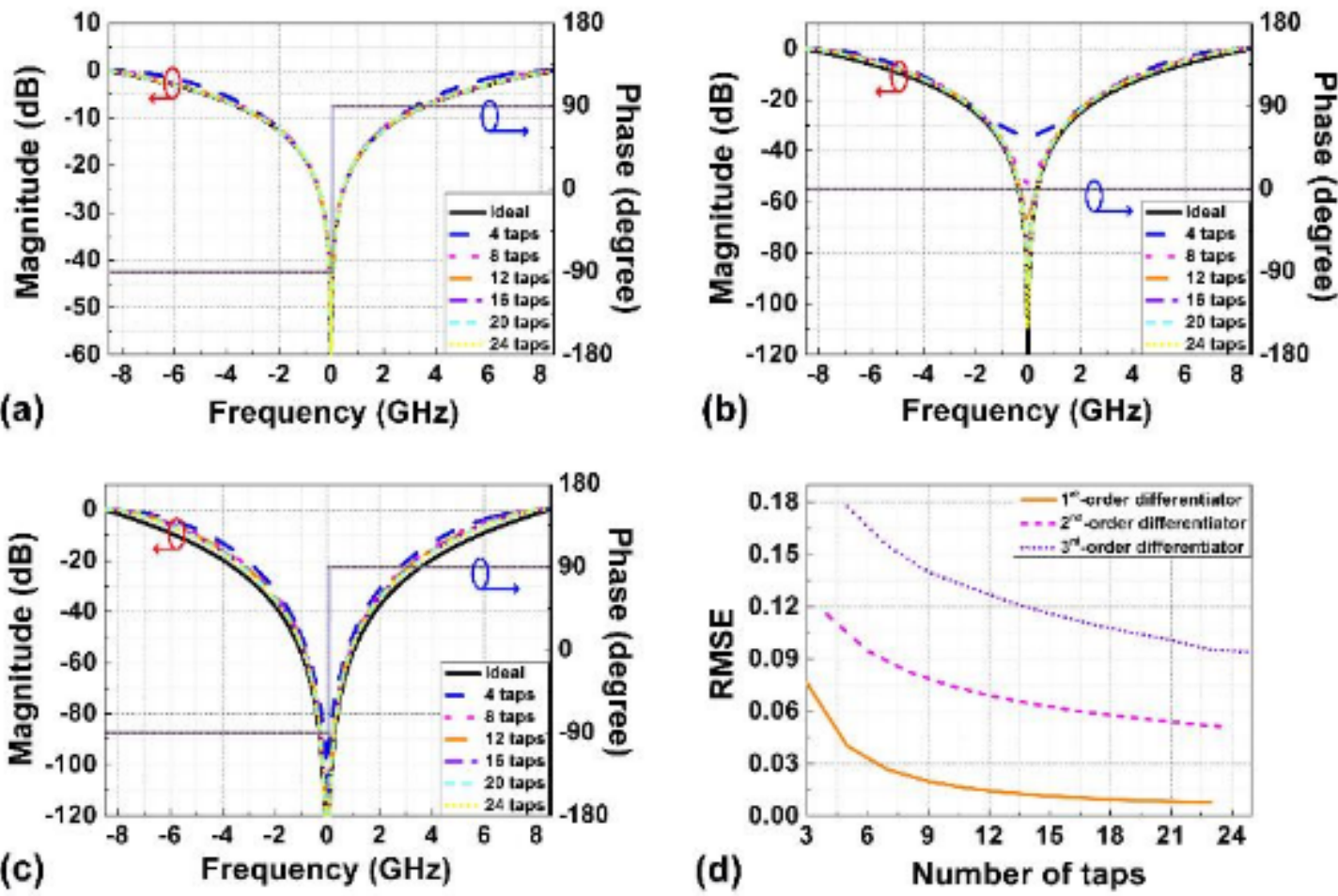

Fig. 1. Simulated RF amplitude and phase responses of the (a) first-, (b) second-, and (c) third-order temporal differentiators. (d) RMSEs between calculated and ideal RF amplitude responses of the first-, second-, and third-order intensity differentiators as a function of the number of taps.

\section{Figure 1}

Simulated RF amplitude and phase responses of the (a) first-, (b) second-, and (c) third-order temporal differentiators. (d) RMSEs between calculated and ideal RF amplitude responses of the first-, second-, and third-order intensity differentiators as a function of the number of taps.

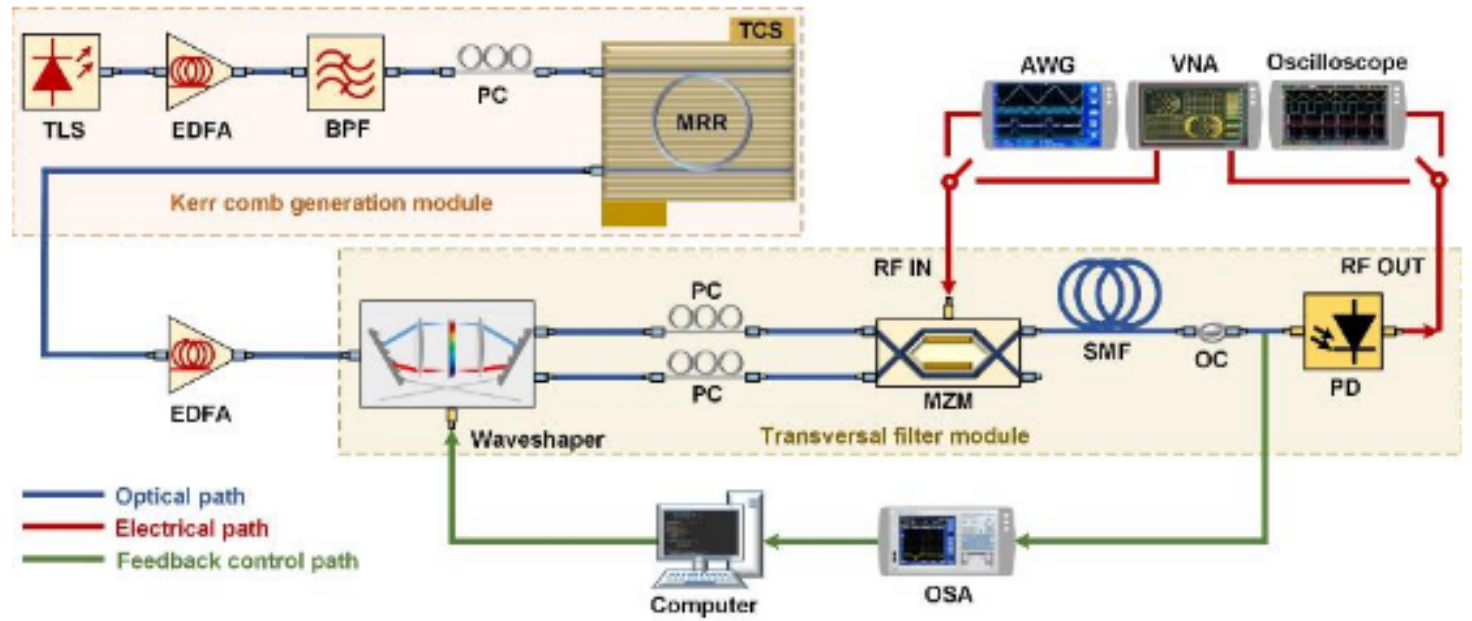

Fig. 2. Schematic illustration of the reconfigurable microwave photonic intensity differentiator. TLS: Tunable laser source. EDFA: erbium-doped fibre amplifier. PC: polarization controller. BPF: optical bandpass filter. TCS: temperature controller stage. MZM: Mach-Zehnder modulator. SMF: single mode fibre. OC: optical coupler. PD: photodetector. OSA: optical spectrum analyzer. VNA: vector network analyzer. AWG: arbitrary waveform generator. 


\section{Figure 2}

Schematic illustration of the reconfigurable microwave photonic intensity differentiator. TLS: Tunable laser source. EDFA: erbium-doped fibre amplifier. PC: polarization controller. BPF: optical bandpass filter. TCS: temperature controller stage. MZM: Mach-Zehnder modulator. SMF: single mode fibre. OC: optical coupler. PD: photodetector. OSA: optical spectrum analyzer. VNA: vector network analyzer. AWG: arbitrary waveform generator.

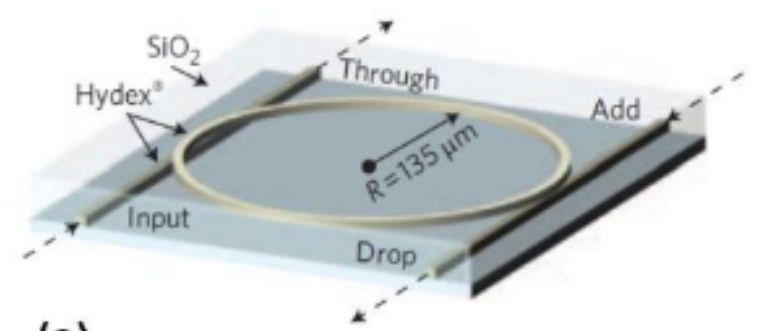

(a)

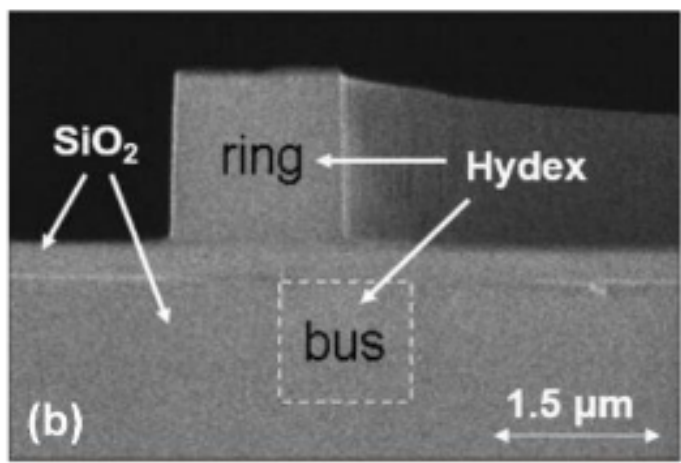

Fig. 3. (a) Hydex MRR. (b) SEM image of the cross-section of the Hydex MRR before depositing the $\mathrm{SiO}_{2}$ upper cladding.

\section{Figure 3}

(a) Hydex MRR. (b) SEM image of the cross-section of the Hydex MRR before depositing the SiO2 upper cladding. 

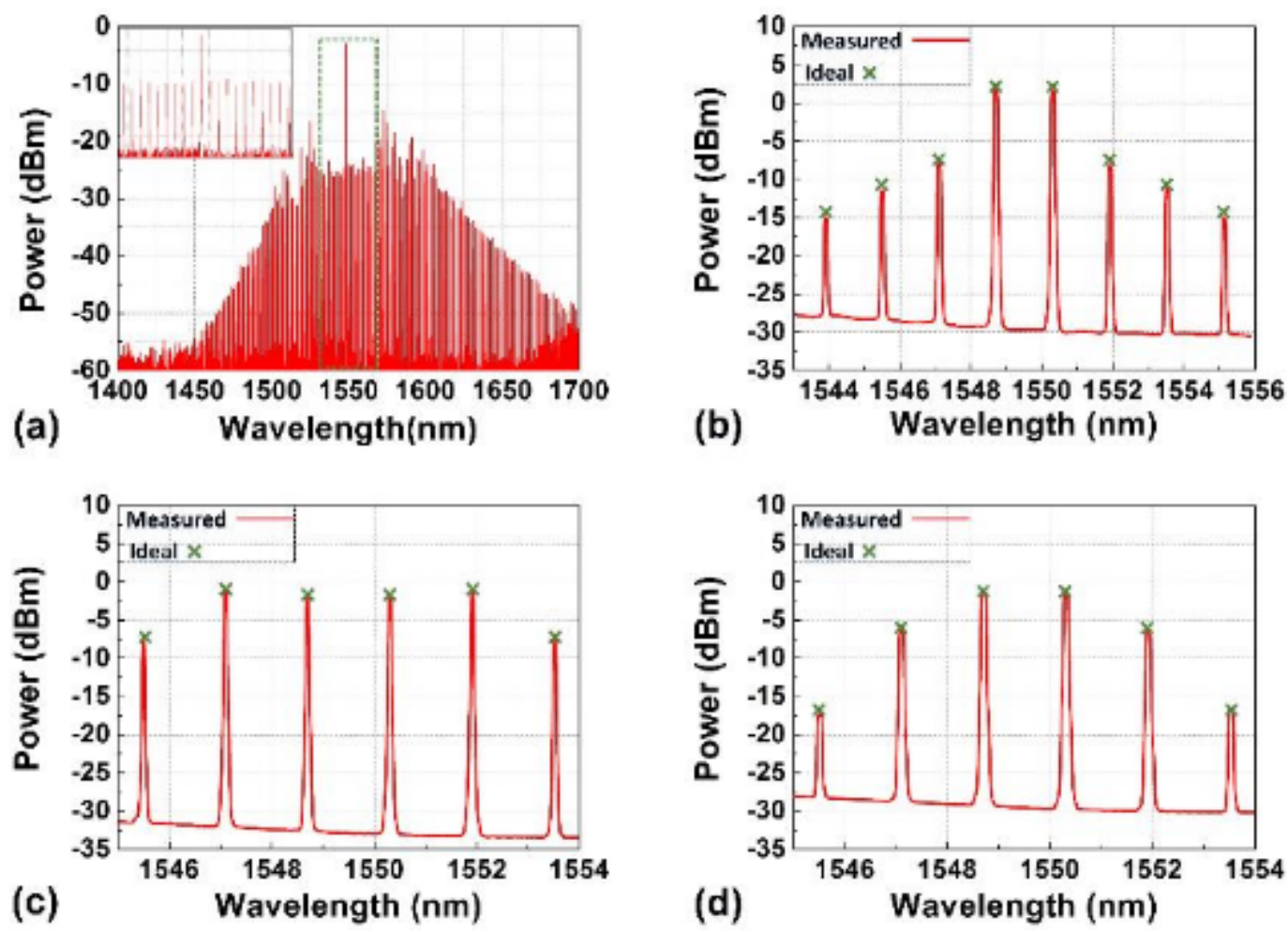

Fig. 4. (a) Optical spectrum of the generated Kerr comb in a 300-nm wavelength range. Inset shows a zoom-in spectrum with a span of $\sim 32 \mathrm{~nm}$. (b)-(d) Measured optical spectra (red solid) of the shaped optical combs and ideal tap weights (green crossing) for the first-, second-r, and thirdorder intensity differentiators.

\section{Figure 4}

(a) Optical spectrum of the generated Kerr comb in a 300-nm wavelength range. Inset shows a zoom-in spectrum with a span of $32 \mathrm{~nm}$. (b)-(d) Measured optical spectra (red solid) of the shaped optical combs and ideal tap weights (green crossing) for the first-, second-r, and third-order intensity differentiators. 


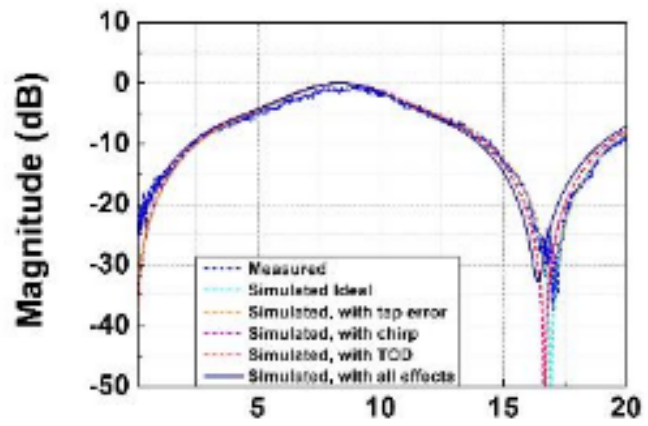

(a-i)

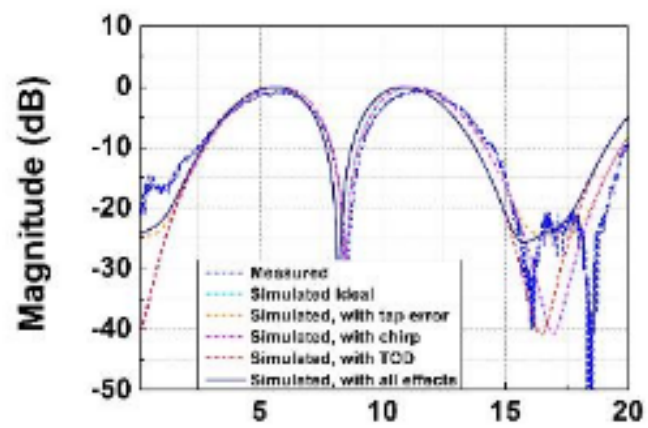

(b-i)

\section{Frequency $(\mathrm{GHz})$}

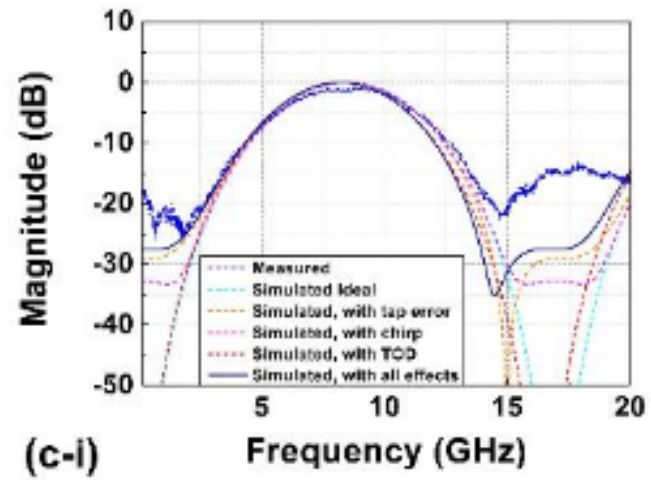

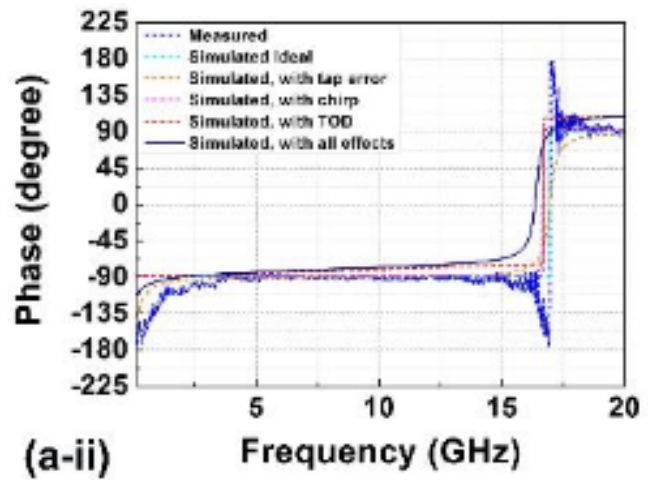

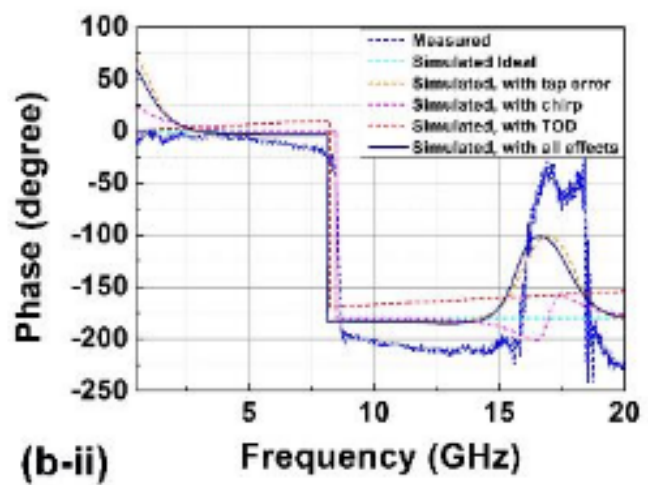

(b-ii) Frequency $(\mathrm{GHz})$

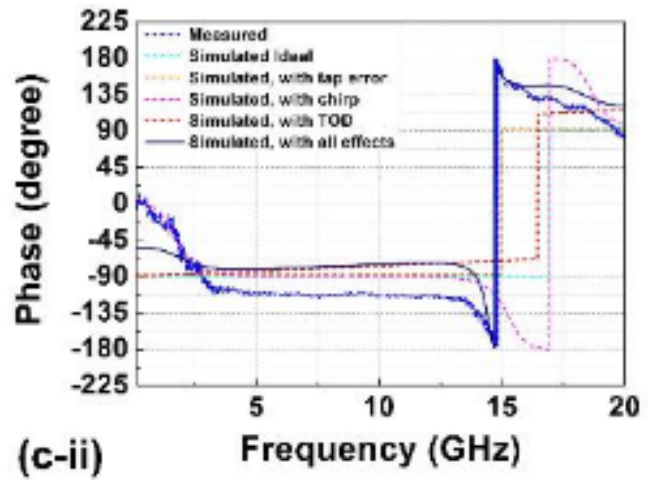

Fig. 5. Measured and simulated RF amplitude and phase responses of (a-i)-(a-ii) the first-order, (b-i)-(b-ii) second-order, and (c-i)-(c-ii) third-order intensity differentiators.

\section{Figure 5}

Measured and simulated RF amplitude and phase responses of (a-i) - (a-ii) the first-order, $(b-i)-(b-i i)$ second-order, and (c-i)-(c-ii) third-order intensity differentiators. 

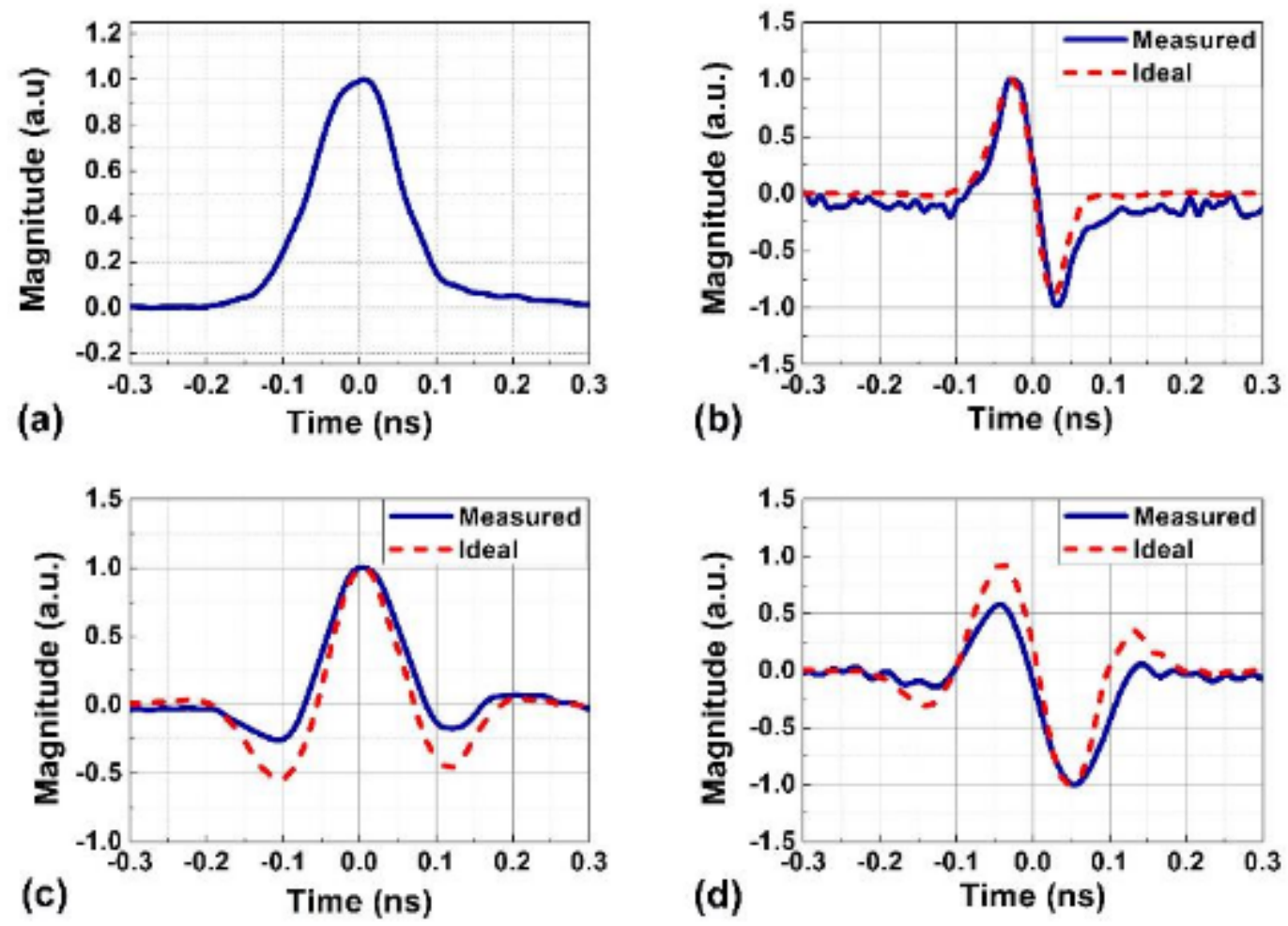

Fig. 6. (a) Measured temporal waveforms of a Gaussian input pulse. Theoretical (red dashed) and experimental (blue solid) responses of the (b) first-, (b) second-, and (c) third-order differentiators.

Figure 6

(a) Measured temporal waveforms of a Gaussian input pulse. Theoretical (red dashed) and experimental (blue solid) responses of the (b) first-, (b) second-, and (c) third-order differentiators. 